\title{
Eggshell-inspired membrane-shell strategy for simultaneously improving the sensitivity and detection range of strain sensors
}

\author{
Hongwei $\mathrm{Li}^{1,4 \dagger}$, Ziting $\operatorname{Tan}^{1 \dagger}$, Liqian Yuan ${ }^{1}$, Jie $\mathrm{Li}^{2}$, Xiaosong Chen ${ }^{2}$, Deyang $\mathrm{Ji}^{2}, \mathrm{Kai}_{\text {Zhang }}{ }^{3}$, \\ Wenping $\mathrm{Hu}^{2}$ and Liqiang $\mathrm{Li}^{1,2^{*}}$
}

\begin{abstract}
The tradeoff between sensitivity and detection range (maximum and minimum stretchability) is a key limitation in strain sensors; to resolve this, we develop an efficient and novel strategy herein to fabricate a highly sensitive and stretchable strain sensor inspired by the membrane-shell structure of poultry eggs. The developed sensor comprises a soft and stretchable surface-grafting polypyrrole (s-PPy) film (acting as the membrane) and a brittle Au film (acting as the shell), wherein both films complement each other at the electrical and mechanical levels. Au forms cracks under strain contributing to its high sensitivity and low detection limit, and s-PPy can bridge Au cracks and increase stretchability which has not been used in strain sensors before. The surfacegrafting strategy not only enhances interface adhesion but also tunes the brittle property of native PPy to render it stretchable. Utilizing the synergetic effect of the membrane-shell complementary structure, the strain sensors achieve ultrahigh sensitivity $\left(>10^{7}\right)$, large stretchability $(100 \%)$, and an ultralow detection limit $(0.1 \%)$, demonstrating significant progress in the field of strain sensors. The membrane-shell (Au/s-PPy)structured strain sensor can successfully detect finger motion, wrist rotation, airflow fluctuation, and voice vibration; these movements produce strain in the range of subtle to marked deformations. Results evidence the ultrahigh performance and bright application prospects of the developed strain sensors.
\end{abstract}

Keywords: strain sensor, detection range, high sensitivity, surface-grafting, membrane-shell

\section{INTRODUCTION}

Stretchable strain sensors have been attracting considerable attention owing to their diverse applications in the fields such as electronic skins [1-8], robotics [9-13], and wearable electronics [14-23]. To achieve high-efficiency monitoring of human activities (breath motion $<1 \%$, joint motions $>55 \%$ ), wearable strain sensors need to be developed with a high sensitivity, large stretchability, and a low detection limit. However, typically, a tradeoff exists between the above three key parameters [22-28]. For example, strain sensors with a wide detection range generally exhibit a moderate or low gauge factor (GF); GF is commonly used to quantify and compare sensitivities of the strain sensors. GF $=\left(\Delta R / R_{0}\right) / \varepsilon$, where $\varepsilon$ is the per unit of applied strain, $\Delta R$ is the resistance change, and $R_{0}$ is the initial resistance $[15,17,22,24]$. Kang et al. [19] demonstrated that crack junction is a highly efficient strategy to realize strain sensors with an ultrahigh GF (>2000), evidencing the wide application potential of crack-based sensors in certain types of applications. However, such sensors generally have a limited detection range due to the total rupture of the conductive metal film under large strains. Thus far, realizing a crack-based strain sensor with high sensitivity, a wide detection range, and a low detection limit has been extremely challenging.

In nature, the eggs of reptiles and birds have a shellmembrane structure acting as a protection layer. This structure includes a brittle inorganic salt (mainly $\mathrm{CaCO}_{3}$ )

\footnotetext{
${ }^{1}$ Advanced Nano-materials Division, Suzhou Institute of Nano-Tech and Nano-Bionics (SINANO), Chinese Academy of Sciences (CAS), Suzhou 215123, China

${ }^{2}$ Tianjin Key Laboratory of Molecular Optoelectronic Sciences, Department of Chemistry, Institute of Molecular Aggregation Science, Tianjin University, Tianjin 300072, China

${ }^{3}$ i-lab, SINANO, CAS, Suzhou 215123, China

${ }^{4}$ International Collaborative Laboratory of 2D Materials for Optoelectronics Science and Technology of Ministry of Education, Institute of Microscale Optoelectronics, Shenzhen University, Shenzhen 518060, China

† These authors contributed equally to this work.

* Corresponding author (email: lilq@tju.edu.cn)
} 
outer shell and a soft inner membrane (mainly fibrin), as shown in Fig. 1a. The outer shell is prone to crack under pressure, but the inner membrane can support the outer shell to some extent and maintain the shape without being broken. The shell-membrane structure and its function in the egg have inspired us to solve the above mentioned drawback of crack-based strain sensors. We hypothesize that, if a soft and stretchable conductive membrane is prepared to support the brittle metal film, the stretching range of the crack-based strain sensor can be enhanced.

Herein, we fabricate a strain sensor comprising a brittle $\mathrm{Au}$ thin film (acting as the shell) and a flexible and stretchable conductive surface-grafting polypyrrole (sPPy) film (acting as the membrane). To the best of our knowledge, such a structure has not been used in strain sensors previously. Au films form microcracks under stretching, which contribute to high sensitivity (Fig. S1a) [19]. The s-PPy film itself exhibits stretchability, which may bridge the cracks under a large strain range and thus contribute to high stretchability (Fig. S1b). On this basis, the membrane-shell (Au/s-PPy) complementary structure enables the strain sensor to achieve a high stretchability of $100 \%$, unprecedented-high sensitivity exceeding $10^{7}$ at $100 \%$ strain, a low limit detection strain of $0.1 \%$ (GF of 9.1), and excellent reproducibility as well as stability. To demonstrate its use and benefit in applications, this membrane-shell structure (Au/s-PPy)-based strain sensor is successfully used in the high-resolution detec- tion of finger motion, which produces subtle deformation, to a wide range of movements including wrist rotation, airflow fluctuation, and voice vibration, which produce marked deformations. Thus, the developed sensor shows excellent performance and widespread application prospects.

\section{EXPERIMENTAL SECTION}

\section{Fabrication of polydimethylsiloxane (PDMS) films}

A polydimethylsiloxane (PDMS) mixture of PDMS elastomer and crosslinker (ratio of 10:1) was manually stirred for $30 \mathrm{~min}$ and subsequently degassed in a vacuum reservoir. The resulting mixture was spin-coated onto a silicon substrate that was pretreated with a self-assembled monolayer (trichloro $(1 \mathrm{H}, 1 \mathrm{H}, 2 \mathrm{H}, 2 \mathrm{H}$-perfluorooctyl)silane), as an anti-adhesion layer for the easy molding of high-quality PDMS substrates, and then placed in a vacuum oven at $70^{\circ} \mathrm{C}$ for $2 \mathrm{~h}$. The thickness of the PDMS film was about $100 \mu \mathrm{m}$.

\section{Fabrication of s-PPy films}

Surface-grafting methods were used to deposit PPy onto the PDMS surface. The details of this process are as follows. First, a surface coupler ( $N$-(3-trimethoxysilylpropyl) pyrrole, Py-silane) was immobilized onto the PDMS substrate surface. A culture dish with PDMS films and $10 \mu \mathrm{L}$ Py-silane solution was placed in a vacuum reservoir, which was maintained at a pressure of $10 \mathrm{~Pa}$ for

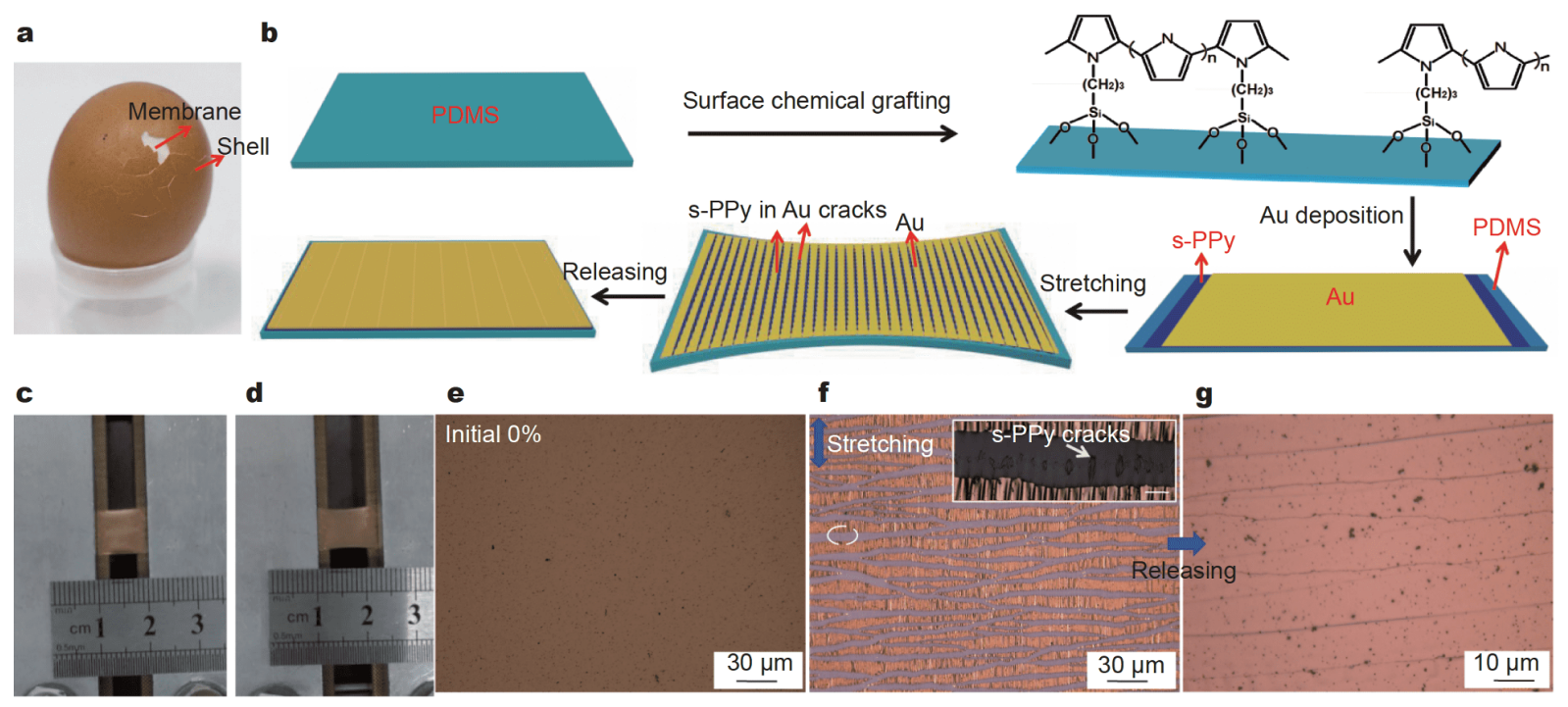

Figure 1 Fabrication procedures and structure of the Au/s-PPy/PDMS strain sensor. (a) Photograph of an egg with some cracks on the brittle outer shell. (b) Fabrication process of the strain sensors based on the synergetic effect of s-PPy and Au thin film. Photographs of the strain sensor at (c) $0 \%$ and (d) 40\%, respectively. Optical images of the surface of Au/s-PPy deposited on PDMS: (e) initial state, (f) stretching at 100\% (the inset is an enlarged image of (f) obtained using confocal laser microscopy), and ( $g$ ) the released state. 
60 min. Second, a conventional chemical oxidation polymerization method was used to synthesize the s-PPy film on the PDMS substrate. To this end, $1 \mathrm{~mL}$ pyrrole aqueous solution with a volume concentration of $3 \%$ was added to $2 \mathrm{~mL}$ of iron(III) $p$-toluenesulfonate hexahydrate aqueous solution with a concentration of $7.8 \mathrm{mg} \mathrm{mL}^{-1}$; then, the Py-silane modified PDMS system was immediately inserted into this mixture solution to allow for a reaction spanning $15 \mathrm{~min}$. This duration is the best time $(t)$ to achieve excellent performance of a strain sensor based on s-PPy/PDMS (Fig. S2). The residual reaction products on the substrates were then ultrasonically washed with deionized water for $\sim 1 \mathrm{~min}$. The concentration of pyrrole and catalyst and the reaction time, the modification of which can change the thickness of PPy [29], are the optimal parameters for this study (Fig. S2).

\section{Fabrication of strain sensors and measurement}

A gold film with the optimal thickness of $15 \mathrm{~nm}$ (the highest performance of the strain sensor based on $\mathrm{Au} / \mathrm{s}$ PPy/PDMS was obtained at this thickness (Fig. S3)) was deposited onto the prepared s-PPy/PDMS structure via thermal evaporation under a pressure of $10^{-4} \mathrm{~Pa}$ at a deposition rate of $0.01 \mathrm{~nm} \mathrm{~s}^{-1}$ to control the adhesion force of s-PPy toward PDMS and Au. After device fabrication, the device needs to be prestrained once before using because the strain characteristics in the first stretching process are generally lower. After the first stretching treatment, the strain characteristics of the device became repeatable and reliable. The purpose of the prestrain treatment is to create cracks in the $\mathrm{Au}$ and sPPy layers and fix the format of the sensor. A laser confocal microscope (Keyence corporation, Japan, Keyence VK-X200) was used to measure the optical images. Electrical characterization of the strain sensor was performed in ambient conditions using a Keithley 4200-SCS (Tektronix company, the United States of America), and the strains were applied using a computer-controlled high-precision stepper motor (Beijing Optical Century Instrument Co., Ltd, SC100, China). The cycling stability test was performed through repeated loading and unloading with different applied strain ranges produced by a computer-controlled stepper motor.

\section{RESULTS AND DISCUSSION}

Fig. 1b schematically illustrates the fabrication process of the membrane-shell structure of the fabricated strain sensor. The PDMS film was fabricated on a silicon wafer. The surface-grafting conductive PPy film was covalently bonded onto PDMS via the in situ chemical oxidation polymerization method (Fig. S4) [29]. Following polymerization, the surface of the s-PPy/PDMS elastomer became macroscopically flat and smooth (Fig. S5). Herein, s-PPy was selected because of its improved stretchability and strong interface adhesion. In practice, most native conductive polymers show brittleness and fracture at low strains (generally below 10\%) [30]. The surface-grafting strategy can tune the brittle property of the native PPy to render it stretchable $(140 \%$ in this work), which has been demonstrated for the first time here. Moreover, conventional conductive polymers generally exhibit poor adhesion on untreated PDMS surfaces. For example, a spin-coated poly(3,4-ethylenedioxythiophene)-poly(styrenesulfonate) (PEDOT:PSS) film on PDMS is prone to peel-off (Fig. S6a, b), rendering it unsuitable for strain sensor applications. In contrast, sPPy shows good interface adhesion toward PDMS (Fig. S6c, d), and during tensile loading, the PDMS substrates firmly fix the films and help delocalize the strain to a network of isolated microcracks (see the inset of Fig. 1f). The stretchability is improved because of the formation of these networks of isolated microcracks that preserve an interconnected network of s-PPy ligaments for electrical conduction. A highly conductive Au thin film was deposited on s-PPy via physical vacuum deposition, which enhanced the GF of the device (for more details, see the text below in this section). Fig. 1c, d and Fig. S7 show actual photographs of the Au/s-PPy/PDMS film sensor.

Fig. 1e-g show optical images of the surface morphology of two-conductive-layer strain sensors. The surface of the $\mathrm{Au}$ film deposited on the s-PPy/PDMS film before stretching showed a smooth and uniform surface without any noticeable undulation. To probe the mechanism of the sensor under mechanical strain, the surface morphology of the Au/s-PPy/PDMS layer was monitored in situ during a stretching/releasing manipulation via optical microscopy. When a uniaxial strain of $50 \%$ was applied to the $\mathrm{Au} / \mathrm{s}-\mathrm{PPy} / \mathrm{PDMS}$ structure, long and straight cracks (about $8 / 100 \mu \mathrm{m}$ on average) perpendicular to the straining direction emerged on the Au film (Fig. S8). The microcracks spontaneously form a uniform pattern to release large stresses. When the Au/s-PPy/PDMS structure was stretched to $100 \%$, more stripe-like cracks (about $11 / 100 \mu \mathrm{m}$ on average) were generated and propagated in the Au layer (Fig. 1f), whereas island-like, smaller cracks form in the s-PPy layer (see the inset of Fig. 1f). These data reveal that $\mathrm{Au}$ and s-PPy form different types of cracks and their cracks do not overlap completely, which is beneficial for high stretchability. The formation of 
different cracks in s-PPy and Au may stem from the following two reasons: (1) the tensile moduli of s-PPy and $\mathrm{Au}$ are different, and (2) the surface-grafting conductive PPy network has strong interfacial interaction between sPPy and PDMS (Figs S5, S6c), resulting in the localization of strain under stress. In contrast, the Au thin film comprises a point-to-point particle network, and the interaction between gold particles is weak. Fig. $1 \mathrm{~g}$ and Fig. S9 exhibit typical morphology of the surface of the $\mathrm{Au} / \mathrm{s}$-PPy/PDMS strain sensor after releasing, in which the linear cracks can be clearly observed.

The working principle of the strain sensor is the change in electrical resistance caused by the formation and propagation of microcrack junctions under mechanical deformation, accompanied by the bridge function of s-PPy over Au film cracks (Fig. 2a and Fig. S1). When the strain sensor was stretched, the formation of cracks and variation of junctions on the Au film resulted in high sensitivity, and the bridge function of the s-PPy and the $\mathrm{Au}$ film was beneficial for high stretchability. To test the sensor's electrical and mechanical properties, the conductive polymer (s-PPy) and metal ultrathin film $(\mathrm{Au})$ bilayer structure were used as strain sensors. Fig. $2 b$ presents excellent linear current-voltage characteristics with different strains. The decrease in the slopes of the current-voltage curves under stretching indicates the increase in resistance, which mainly stems from the for- mation, propagation, and broadening of cracks. Significantly, Fig. $2 \mathrm{c}$ shows that the variation in relative resistance is substantial over several orders of magnitude with an applied strain of about $100 \%$, thereby producing high sensitivity and primarily originating from the synergetic effect of the s-PPy/Au membrane-shell complementary structure. The cycle test indicates that there is small hysteresis, particularly in the large strain range between the stretching and releasing process (Fig. 2c). This may be due to the viscoelasticity of PDMS elastomer as well as the imperfect disconnection-reconnection of the $\mathrm{Au}$ and s-PPy cracks, which is commonly observed in strain sensors [15,24,31-33].

To demonstrate the superiority of the membrane-shell complementary structure (Au/s-PPy/PDMS), stretchable $\mathrm{Au} / \mathrm{PDMS}$ and s-PPy/PDMS were fabricated and tested for comparison, as shown in Fig. 2d, e. A relative resistance change exceeding $10^{4}$ and a GF of about $5.6 \times 10^{5}$ at a strain of $6 \%$ were observed for Au/PDMS, but the critical (or maximum) strain was as small as $\sim 6.5 \%$. This indicates that the $\mathrm{Au} / \mathrm{PDMS}$ sensors in the absence of an s-PPy layer possess high sensitivity but low stretchability. This phenomenon is mainly attributed to the formation of a continuous crack in the Au film that completely cuts off the conductive channel under large stretching (Fig. S1). In contrast, s-PPy/PDMS possessed a high strain of $100 \%$ with the resistance change and GF both
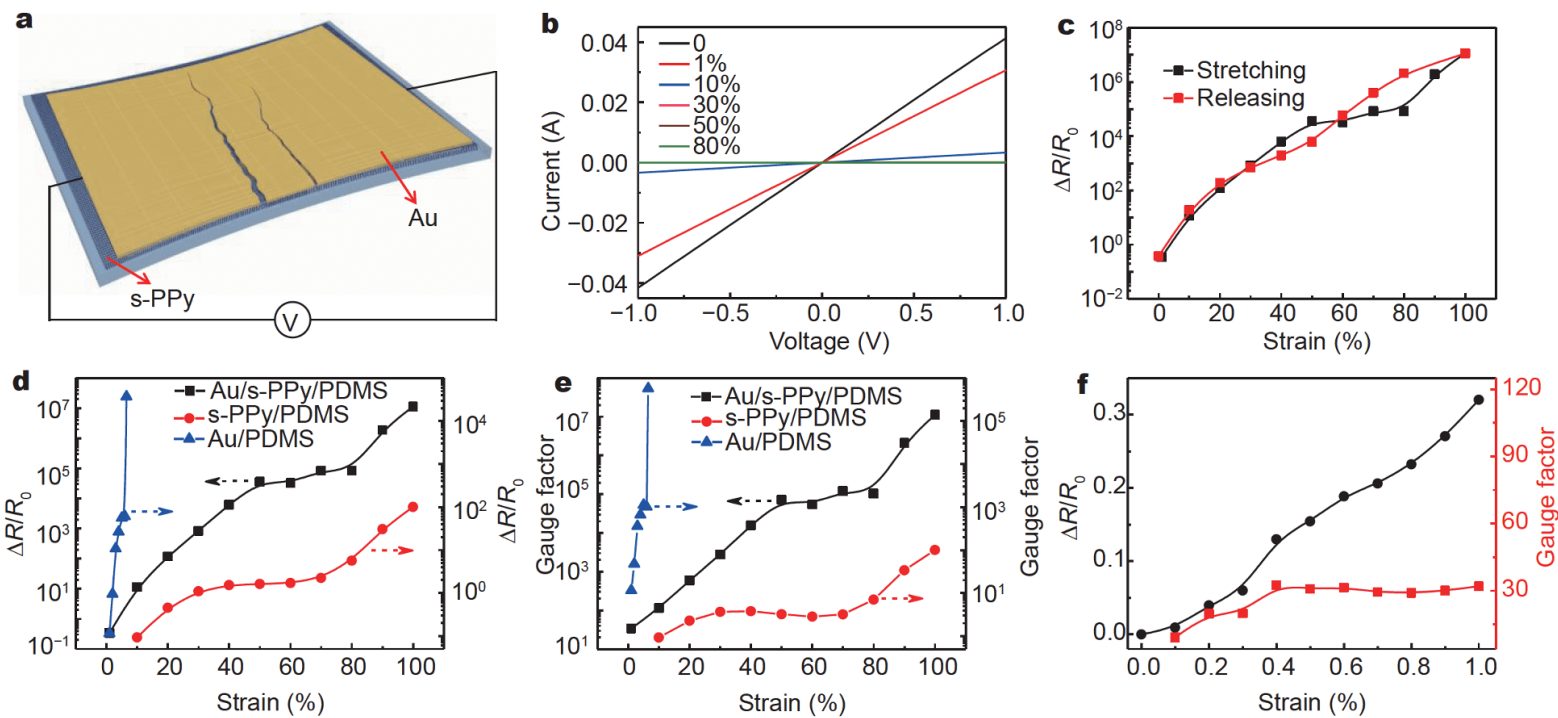

Figure 2 Working principle and sensing properties of the Au/s-PPy/PDMS strain sensor. (a) Schematic model of the basic circuit of the sensors. (b) Current-voltage characteristics of the Au/s-PPy/PDMS strain sensors. (c) The curves of relative resistance variation vs. applied strain of the Au/s-PPy/ PDMS strain sensors under the stretching and releasing process. (d) Resistances responses with increasing strain for the device of Au/s-PPy/PDMS (0$100 \%$ ), s-PPy/PDMS (0-100\%), and Au/PDMS (0-6.5\%). (e) Strain sensor performances of the Au/s-PPy/PDMS, s-PPy/PDMS, and Au/PDMS. (f) Relative resistance variation $v s$. applied strain in the range of $0.1 \%-1 \%$. 
being 385 (Fig. 2d, e). This result indicates that surface grafting provides an efficient and novel approach to render the brittle native conductive polymer stretchable, which is different from the conventional methods that blend additives such as plasticizers or other polymers into the structure [30]. This is mainly because surface grafting localizes the stress and provides numerous sites for crack nucleation inducing the island-like crack formation (the inset in Fig. 1f).

The above results indicate that the exclusive use of pure $\mathrm{Au}$ or s-PPy can realize high GF or large stretchability. Remarkably, when combining Au and s-PPy together as an active layer, the GF can reach a high level of $2.4 \times 10^{7}$ at a large strain of $100 \%$ (Fig. 2e), which has never been realized before. The ultrahigh sensitivity in our work is attributed to the synergetic effect of the membrane-shell complementary structure (Au and s-PPy), which has the following three merits. (1) Au and s-PPy have different tensile moduli and thus can form different cracks during stretching (Fig. 1f). (2) Under small strains, cracks form on the Au film and quickly propagate with strain, which is beneficial for the high sensitivity of the strain sensor (for details, see Fig. S1) [19]. (3) s-PPy can be used as a conductive bridge for Au film cracks that constructs an effective percolation network (for detailed evidence, see Movie S1 and Figs S10, S11), which endows the Au/s-PPy double layers with certain electrical conductivity (the resistivity of s-PPy $<15 \Omega \mathrm{cm}$ ) under high strains and thus contributes to the high stretchability.

Additionally, Fig. 2c-e show three typical, different regions in the strain characteristics of the Au/s-PPy/ PDMS sensor, which can be attributed to nonuniform crack propagation and broadening (Fig. S1) in the Au and s-PPy film during the stretching and releasing process. In practice, the nonlinear dependence of relative resistance variation on strain, which results in strain-dependent GF, is commonly observed in crack-based sensors $[17,19,20,34]$ and other strain sensors $[11,35]$. This may be due to the nonlinear change in the conductive route during the stretching process.

To further demonstrate the high sensitivity of our unique strain sensor, a subtle change in strain was detected, because the high sensitivity of strain sensor indicated that it could be used as an ultrasensitive strain sensor to detect subtle change in strain. Fig. $2 \mathrm{f}$ presents the relative resistance variation and GF $v s$. applied strain $(<1 \%)$, and Fig. S12 shows the stepped curves of the relative resistance $v$ s. applied strain $(<1 \%)$. Remarkably, the detection limit and strain resolution are both as small as $0.1 \%$, definitely confirming the ultrasensitive property of the fabricated sensors. GF was calculated to be 9.1 at a strain of $0.1 \%$, which is considered very good performance for such a small strain. This result indicates that the fabricated sensor can be used for the ultrasensitive detection of small strains.

To investigate the reproducibility of the high sensitivity and stretchability of the Au/s-PPy/PDMS strain sensors, 15 sensors were fabricated. As shown in Fig. 3a, 60\% sensors could stretch to $100 \%$, and $1 / 3$ of the total sensors produced GF $>10^{7}$; another $1 / 3$ of the sensors produced GF $>10^{6}$. To compare the performance of our strain sensor with that of reported devices [24,31-33,35-42], a three-dimensional graph of GF, detection limit, and maximum strain is presented in Fig. 3b, and more specifications are listed in Table S1. Analyzing the data in Fig. $3 \mathrm{~b}$ and Table S1, the following can be deduced. (1) At a high stretchability of $100 \%$, the GF of the Au/s-PPy/ PDMS strain sensor is $>10^{6}$ higher than the best value reported in literature. In addition, the detection limit $(0.1 \%)$ obtained herein is lower than the value $(0.2 \%)$ reported in literature [31]. (2) At a GF level of $10^{7}$, our sensor is more stretchable (100\%) than the sensor reported (50\%) [32]. (3) At the same GF and stretchability level, our detection limit is as small as $0.1 \%$. Accordingly, our sensor based on the membrane-shell complementary structure realizes the simultaneous improvement of sensitivity, stretchability, and detection limit. To test the repeatability of the strain sensor, dynamic and multiple loading-unloading strain measurements were performed under various strain loading conditions. As shown in Fig. $3 c$, four different stretching strains $(10 \%, 30 \%$, and $80 \%$ ), with 10 cycles for each strain, were applied to the strain sensor. The $R / R_{0}$ value increased with almost complete reversibility. These results indicate the good reproducibility of the fabricated strain sensor.

The dynamic durability of strain sensors represents the endurance of the device toward long-term stretching/releasing cycles with stable electrical functionality and is crucial in the development of skin-mountable and wearable strain sensors [43]. Thus, the strain was repeatedly varied from 0 to $50 \%$ over 1000 cycles (Fig. 3d, Movie S2); further, the resistance changes were nearly constant, revealing that the crack "contact-separation" and "contactrecovery" process under the loading-unloading cycles exhibits excellent stability and reliability. We also tested the sensing performance after the 10th and 1000th cycles' test (Fig. S13), which remained nearly unchanged. Additionally, the resistance of releasing to the original state only changed a few ohms after stretching to $100 \%$ ten times (over $100 \mathrm{~s}$ ) and removing the strain (Fig. S14). All 

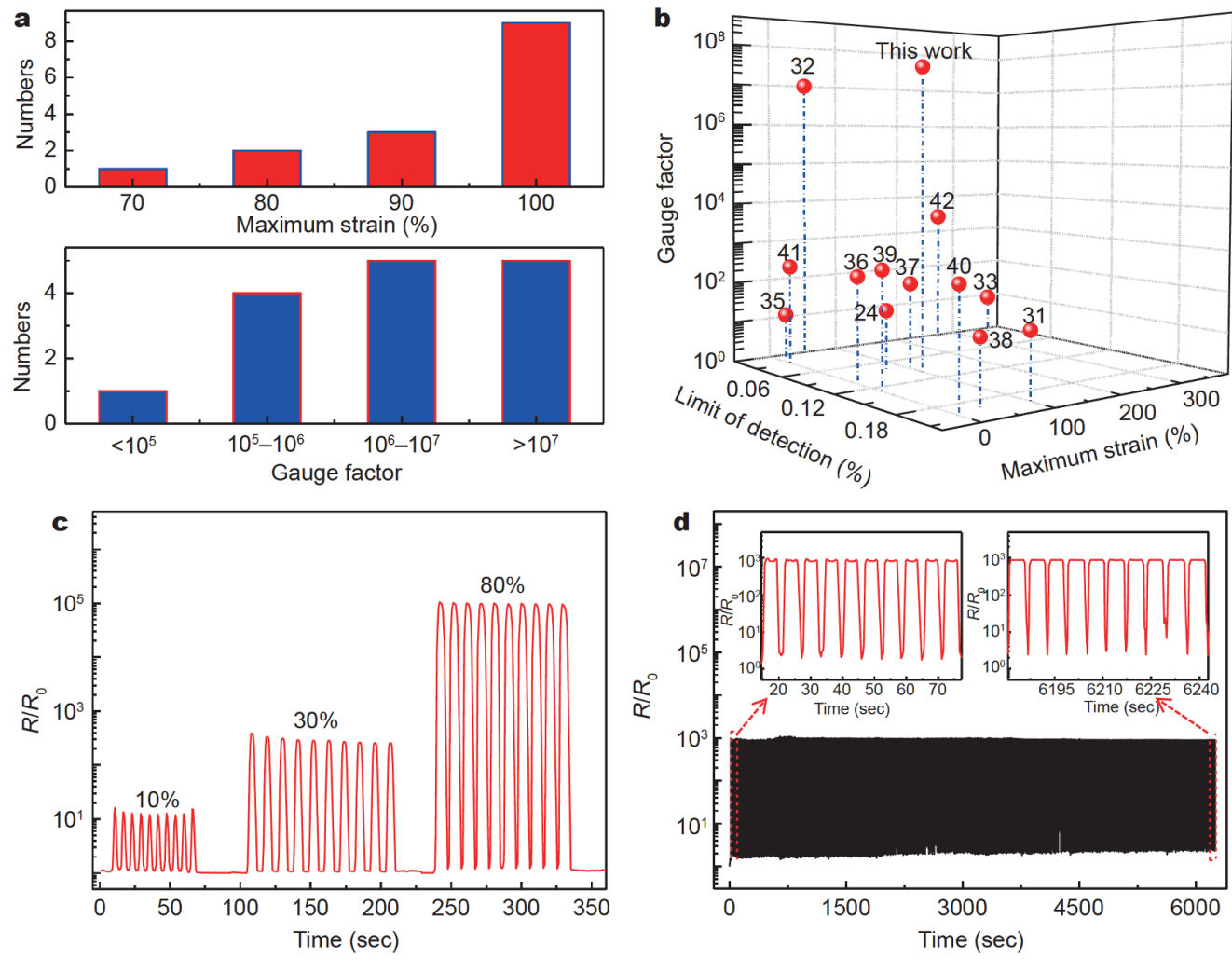

Figure 3 Reproducibility and reliability property of the Au/s-PPy/PDMS strain sensor. (a) Maximum strain (top panel) and GF (bottom panel) distribution for 15 sensors. (b) GF, detection limit, and maximum strain of recent reports and our work. The GF at a strain of $100 \%$ in this work is several orders of magnitude higher than the best value available in literature. (c) The performance of the strain sensor under stretching/releasing cycles of strains of $10 \%, 30 \%$, and $80 \%$. (d) The relative resistance variation under repeated loading and unloading of $50 \%$ strain of 1000 cycles.

these results demonstrate the excellent durability and repeatability of the fabricated strain sensor.

All the above characterizations of high sensitivity, low limit detection, good reliability, high strain resolution, stability, and easy fabrication demonstrate that the strain sensor can be used in various applications such as e-skins and wearable electronics [42]. To investigate the potential application in the field of wearable electronics, the strain sensor was attached to a human forefinger with adhesive tapes for testing the ability of the strain sensor to detect human motion (Fig. 4 and Movie S3). Fig. 4a exhibits the pictures of fingers with different bending degrees, which produce a clear stepped relative resistance change (Fig. 4b). This indicates that the fabricated strain sensor could well recognize the motion of the finger joint. Fig. $4 \mathrm{c}$ displays the cycle test between a relaxed (straightened finger) and stretched (bent finger) state. Upon bending the finger, the strain sensor responds rapidly and repeatedly, and the high signal-to-noise ratios are well maintained. The results indicate that the fabricated strain sensor can be used to steadily monitor human motions.
To demonstrate the low limit detection application of this strain sensor, subtle human motion was detected by attaching a strain sensor to a human forefinger with adhesive tapes (Fig. 4d, e, Fig. S15, and Movie S4). Fig. 4d, e show the changes in signals, which were measured with respect to the subtle bending angle. Benefiting from the high sensitivity of the unique strain sensors, they not only have a wide detection range but also attain an extremely small detection angle, about $0.9^{\circ}$, as displayed in Fig. $4 \mathrm{e}$ and Fig. S15, which is better than, or at least comparable to that of the reported strain sensors [37].

To demonstrate the wide applications of the fabricated strain sensor, wrist rotation, airflow fluctuation by breath, and voice vibration were measured with our sensor as shown in Fig. 4f-h and Movies S5, S6. Fig. 4f and Movie S5 present the detection of the wrist rotation using the fabricated strain sensor, showing a clear sensing signal (resistance change). This result, together with the above finger motion test, indicates that our sensor can be used to monitor various human motions.

In addition to monitoring human motion, our sensor 

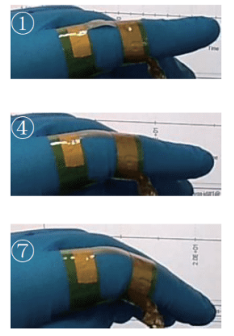
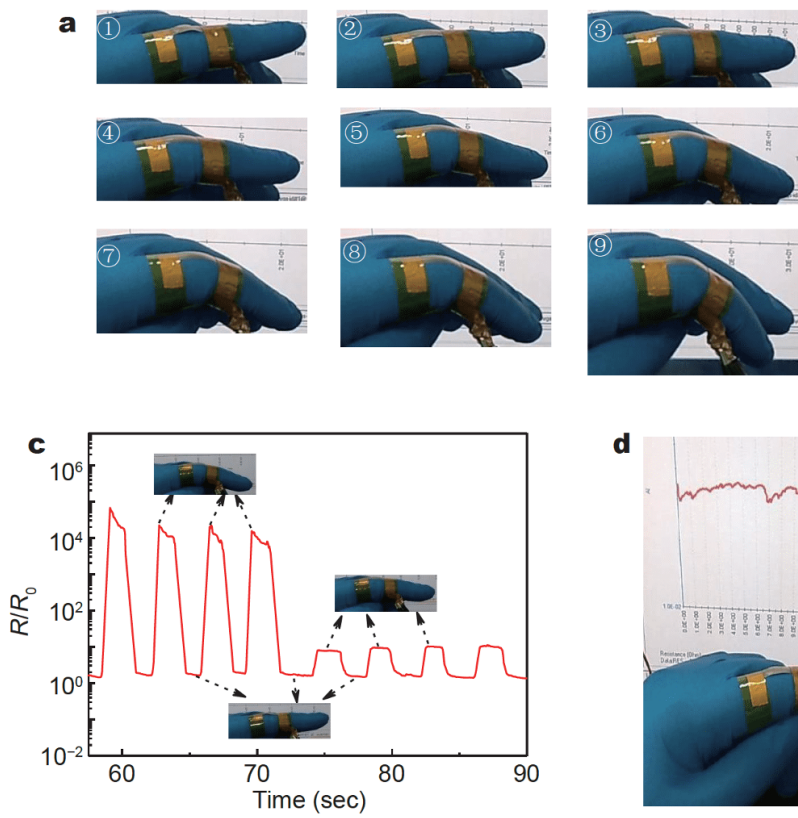

d
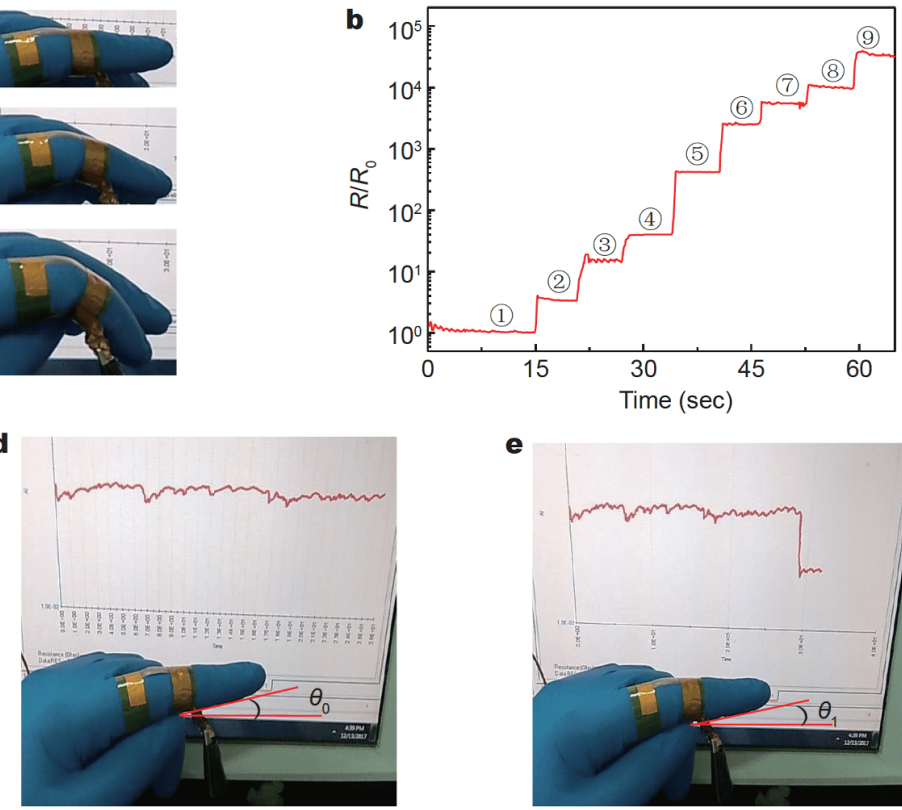

e
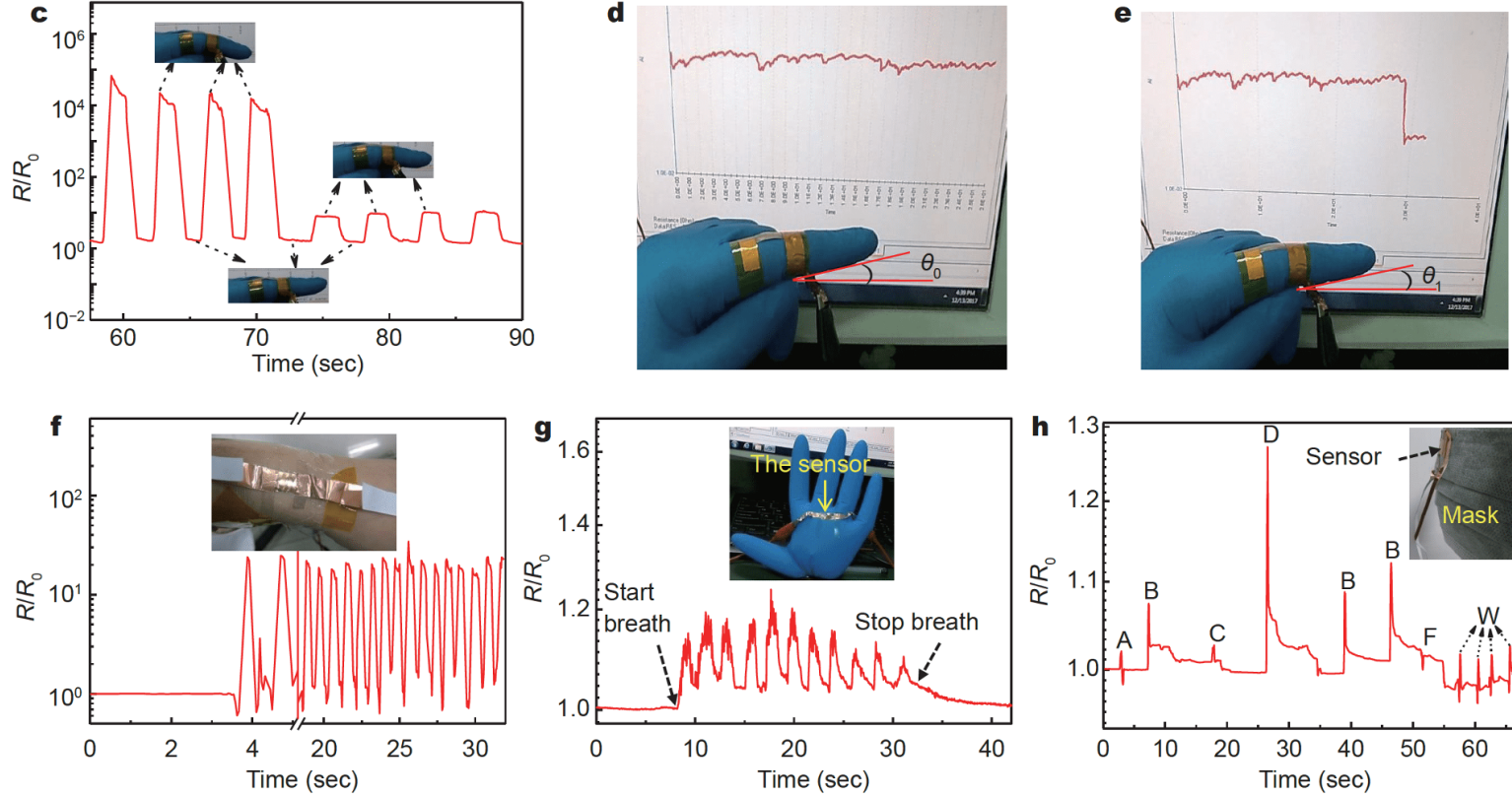

Figure 4 Exemplary applications of the Au/s-PPy/PDMS strain sensors. Human motion detection by attaching the strain sensor onto the forefinger. (a) Photographs of a strain sensor tied onto a forefinger bending at different degrees. (b) Resistance change of the sensor response to (a). (c) Resistance change curve during repeated relaxing and bending of the forefinger. (d, e) Detection of slight human motion using the Au/s-PPy/PDMS strain sensor. Bending of the forefinger to about (d) $0^{\circ}$ and (e) $0.9^{\circ}$. (f) Detection of human wrist rotation. (g) Tiny airflow fluctuation caused by breathing toward the sensor. (h) Human voice detection by attaching the strain sensor onto a mask.

can be used to detect the strain induced by other mechanical stimuli such as airflow fluctuation and voice vibration. Fig. 4g and Movie S6 show the detection of the airflow of a human's breath using the fabricated strain sensor that was placed on an inflated glove. The flow of breath toward the sensor causes a strain within a small range, but produces a clear and repeatable sensing signal. This result shows the application potential of our sensor in the detection of weak airflow.

As shown in Fig. 4h, the strain sensor was placed on the mask. When the person spoke different English letters of the alphabet, a clear sensor signal could be detected. In practice, voice vibration produces a very weak deformation in the strain sensor attached on the mask, and the strain range is $<1 \%$ by geometric estimation with center mark method. Remarkably, the different letters generate distinctive signals and the same letter produces similar signals, evidencing the excellent performance of the fabricated sensor and suggesting its potential application in voice recognition. All these experiments demonstrate the wide application potential of the Au/s-PPy/PDMS strain sensor.

Above all, the membrane-shell structure enables the strain sensor to achieve high sensitivity, low detection limit, high stretchability, and good stability, which can be understood as follows. (1) Au and s-PPy have high and low conductivity, respectively; hence, the resistance of the $\mathrm{Au} / \mathrm{s}$-PPy sensor has the capacity to change over a large range (several orders of magnitude), which is the basis for high sensitivity (GF). (2) Au is prone to form cracks and 
produces large resistance change, which is beneficial for the characteristics of high sensitivity and low detection limit (Fig. S1) [19]. Further, s-PPy forms smaller and fewer cracks under a larger mechanical strain range, differing from native brittle conductive polymer. Therefore, s-PPy can bridge the cracked Au film, which is beneficial for large stretchability (Fig. S1). (3) s-PPy shows strong adhesion to PDMS and Au (Fig. S6c, d), thereby improving the device stability $[29,44]$. From the perspective of structure and function, such a membraneshell complementary bilayer structure and the use of sPPy material have not been reported in the field of strain sensors. Most conventional sensors adopt a bulk network composite structure [25,27], which is unfavorable to simultaneously achieving high sensitivity, large strain range, and low detection limit, because it is difficult for bulk composite structures to attain large resistance variation from an ultralow to large strain range. In contrast, the planar membrane-shell bilayer structure can achieve the above target because its two layers can work separately in the ultralow and large strain range with high sensitivity.

\section{CONCLUSIONS}

In this work, to resolve the key issue of tradeoff among sensitivity, stretchability, and detection limit in the field of strain sensors, we developed an ultrasensitive and stretchable strain sensor based on the membrane-shell complementary structure. In this structure, $\mathrm{Au}$ (brittle shell) and s-PPy (soft membrane) complement each other at the electrical and mechanical levels. Consequently, this structure provides the strain sensor with a maximum strain of $100 \%$, a minimum strain of $0.1 \%$, an ultrahigh sensitivity of $>10^{7}$, and excellent reliability as well as stability. Furthermore, benefiting from the combination of high sensitivity, high stretchability, and low detection limit, the strain sensor can detect human motions, airflow fluctuation, and voice vibration, which produce strains from a small to a wide range of strain. Therefore, this unique membrane-shell complementary structure of conductive bilayer ( $\mathrm{Au}$ and s-PPy) provides a new and efficient strategy to construct high-performance strainmonitoring devices for next-generation flexible and wearable electronic systems.

Received 28 May 2020; accepted 23 July 2020;

published online 20 August 2020

1 Gibney E. The inside story on wearable electronics. Nature, 2015, 528: $26-28$

2 Kim J, Lee M, Shim HJ, et al. Stretchable silicon nanoribbon electronics for skin prosthesis. Nat Commun, 2014, 5: 5747-5758

3 Lee TI, Jang WS, Lee E, et al. Ultrathin self-powered artificial skin. Energy Environ Sci, 2014, 7: 3994-3999

4 Stingelin-Stutzmann N. Complexity made simple. Nat Mater, 2008, 7: $171-172$

5 Tee BCK, Wang C, Allen R, et al. An electrically and mechanically self-healing composite with pressure- and flexion-sensitive properties for electronic skin applications. Nat Nanotech, 2012, 7: 825832

6 Wu X, Peng H. Polymer-based flexible bioelectronics. Sci Bull, 2019, 64: 634-640

7 Bian J, Zhou L, Wan X, et al. Laser transfer, printing, and assembly techniques for flexible electronics. Adv Electron Mater, 2019, 5: 1800900

8 Zheng Z, Ni Z, Zhang X, et al. Carbon nanotubes assisting interchain charge transport in semiconducting polymer thin films towards much improved charge carrier mobility. Sci China Mater, 2019, 62: 813-822

9 Yao L, Cheng T, Shen X, et al. Paper-based all-solid-state flexible asymmetric micro-supercapacitors fabricated by a simple pencil drawing methodology. Chin Chem Lett, 2018, 29: 587-591

10 Wang B, Huang W, Chi L, et al. High- $k$ gate dielectrics for emerging flexible and stretchable electronics. Chem Rev, 2018, 118: 5690-5754

11 Amjadi M, Pichitpajongkit A, Lee S, et al. Highly stretchable and sensitive strain sensor based on silver nanowire-elastomer nanocomposite. ACS Nano, 2014, 8: 5154-5163

12 Kan J, Wang S, Wang Z, et al. Fabrication of flexible thin organic transistors by trace water assisted transfer method. Chin Chem Lett, 2018, 29: 1681-1684

13 Kim KK, Hong S, Cho HM, et al. Highly sensitive and stretchable multidimensional strain sensor with prestrained anisotropic metal nanowire percolation networks. Nano Lett, 2015, 15: 5240-5247

14 Yang M, Zhao X, Tang Q, et al. Stretchable and conformable synapse memristors for wearable and implantable electronics. Nanoscale, 2018, 10: 18135-18144

15 Li W, Guo J, Fan D. 3D graphite-polymer flexible strain sensors with ultrasensitivity and durability for real-time human vital sign monitoring and musical instrument education. Adv Mater Technol, 2017, 2: 1700070

16 Tang D, Wang Q, Wang Z, et al. Highly sensitive wearable sensor based on a flexible multi-layer graphene film antenna. Sci Bull, 2018, 63: 574-579

17 Liu Z, Qi D, Guo P, et al. Thickness-gradient films for high gauge factor stretchable strain sensors. Adv Mater, 2015, 27: 6230-6237

18 Dong H, Yan Q, Hu W. Multilevel investigation of charge transport in conjugated polymers-new opportunities in polymer electronics. Acta Polymerica Sinica, 2017, 8: 1246-1260

19 Kang D, Pikhitsa PV, Choi YW, et al. Ultrasensitive mechanical crack-based sensor inspired by the spider sensory system. Nature, 2014, 516: 222-226

20 Park B, Kim J, Kang D, et al. Dramatically enhanced mechanosensitivity and signal-to-noise ratio of nanoscale crack-based sensors: effect of crack depth. Adv Mater, 2016, 28: 8130-8137

21 Zhang JL, Han C, Hu Z, et al. 2D phosphorene: epitaxial growth and interface engineering for electronic devices. Adv Mater, 2018, 30: 1802207

22 Liao X, Zhang Z, Kang Z, et al. Ultrasensitive and stretchable resistive strain sensors designed for wearable electronics. Mater Horiz, 2017, 4: 502-510 
23 Li L, Lou Z, Chen $\mathrm{H}$, et al. Stretchable $\mathrm{SnO}_{2}-\mathrm{CdS}$ interlacednanowire film ultraviolet photodetectors. Sci China Mater, 2019, 62: 1139-1150

24 Jeong YR, Park H, Jin SW, et al. Highly stretchable and sensitive strain sensors using fragmentized graphene foam. Adv Funct Mater, 2015, 25: 4228-4236

25 Zhao S, Guo L, Li J, et al. Binary synergistic sensitivity strengthening of bioinspired hierarchical architectures based on fragmentized reduced graphene oxide sponge and silver nanoparticles for strain sensors and beyond. Small, 2017, 13: 1700944

26 Yan C, Wang J, Kang W, et al. Highly stretchable piezoresistive graphene-nanocellulose nanopaper for strain sensors. Adv Mater, 2014, 26: 2022-2027

27 Yang Z, Wang DY, Pang Y, et al. Simultaneously detecting subtle and intensive human motions based on a silver nanoparticles bridged graphene strain sensor. ACS Appl Mater Interfaces, 2018, 10: 3948-3954

$28 \mathrm{Wu} \mathrm{X}, \mathrm{Du}$ R, Fang L, et al. Grain size adjustion in organic fieldeffect transistors for chemical sensing performance improvement. Sci China Mater, 2019, 62: 138-145

29 Li H, Wu K, Xu Z, et al. Ultrahigh-sensitivity piezoresistive pressure sensors for detection of tiny pressure. ACS Appl Mater Interfaces, 2018, 10: 20826-20834

30 Kayser LV, Lipomi DJ. Stretchable conductive polymers and composites based on PEDOT and PEDOT:PSS. Adv Mater, 2019, 31: 1806133

31 Cheng Y, Wang R, Sun J, et al. A stretchable and highly sensitive graphene-based fiber for sensing tensile strain, bending, and torsion. Adv Mater, 2015, 27: 7365-7371

32 Zhou J, Yu H, Xu X, et al. Ultrasensitive, stretchable strain sensors based on fragmented carbon nanotube papers. ACS Appl Mater Interfaces, 2017, 9: 4835-4842

33 Gong S, Lai DTH, Su B, et al. Highly stretchy black gold E-skin nanopatches as highly sensitive wearable biomedical sensors. Adv Electron Mater, 2015, 1: 1400063

34 Chortos A, Lim J, To JWF, et al. Highly stretchable transistors using a microcracked organic semiconductor. Adv Mater, 2014, 26: 4253-4259

35 Bae SH, Lee Y, Sharma BK, et al. Graphene-based transparent strain sensor. Carbon, 2013, 51: 236-242

36 Zhao J, He C, Yang R, et al. Ultra-sensitive strain sensors based on piezoresistive nanographene films. Appl Phys Lett, 2012, 101: 063112

37 Liu Q, Chen J, Li Y, et al. High-performance strain sensors with fish-scale-like graphene-sensing layers for full-range detection of human motions. ACS Nano, 2016, 10: 7901-7906

38 Wang Y, Wang L, Yang T, et al. Wearable and highly sensitive graphene strain sensors for human motion monitoring. Adv Funct Mater, 2014, 24: 4666-4670

39 Liao X, Liao Q, Yan X, et al. Flexible and highly sensitive strain sensors fabricated by pencil drawn for wearable monitor. Adv Funct Mater, 2015, 25: 2395-2401

40 Zhao J, Wang G, Yang R, et al. Tunable piezoresistivity of nanographene films for strain sensing. ACS Nano, 2015, 9: 1622-1629

41 Coskun MB, Akbari A, Lai DTH, et al. Ultrasensitive strain sensor produced by direct patterning of liquid crystals of graphene oxide on a flexible substrate. ACS Appl Mater Interfaces, 2016, 8: 2250122505

42 He T, Lin C, Shi L, et al. Through-layer buckle wavelengthgradient design for the coupling of high sensitivity and stretch- ability in a single strain sensor. ACS Appl Mater Interfaces, 2018, 10: 9653-9662

43 Amjadi M, Kyung KU, Park I, et al. Stretchable, skin-mountable, and wearable strain sensors and their potential applications: a review. Adv Funct Mater, 2016, 26: 1678-1698

44 Wang S, Wang Z, Li J, et al. Surface-grafting polymers: from chemistry to organic electronics. Mater Chem Front, 2020, 4: 692714

Acknowledgements The authors are grateful to the National Key Research and Development Program (2018YFA0703200 and 2016YFB0401100), the National Natural Science Foundation of China (21573277, 51503221 and 21905199), Tianjin Natural Science Foundation (19JCJQJC62600 and 194214030036), and the Key Research Program of Frontier Sciences of Chinese Academy of Sciences (QYZDB -SSW-SLH031).

Author contributions Li $\mathrm{H}$ performed the fabrication, measurements and mechanism analysis of the strain sensor. Tan Z, Yuan L assisted in the experiments. $\mathrm{Li} \mathrm{L}, \mathrm{Li} \mathrm{H}$ and Tan $\mathrm{Z}$ analyzed the data and wrote the manuscript. Li L conceived and supervised this work. Hu W, Zhang K, Ji $\mathrm{D}$, Chen $\mathrm{X}$ and $\mathrm{Li} \mathrm{J}$ provided valuable and constructive suggestions.

Conflict of interest The authors declare no conflict of interest.

Supplementary information Experimental details and supporting data are available in the online version of the paper.

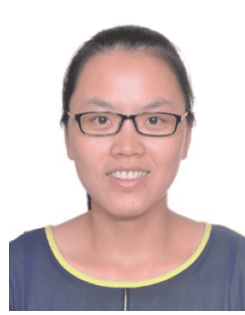

Hongwei $\mathbf{L i}$, is currently a postdoctoral research fellow at the Collaborative Innovation Center for Optoelectronic Science \& Technology of Shenzhen University and i-Lab of Suzhou Institute of Nano-Tech and Nano-Bionics, the Chinese Academy of Sciences (CAS). She obtained her $\mathrm{PhD}$ from Beijing University of Technology (2015), and worked as a postdoctoral research associate at the Advanced Nano-materials Division, Suzhou Institute of Nano-Tech and NanoBionics (SINANO), CAS (2016-2018). Her current research interest focuses on the photodetectors and strain sensors based on 2D materials and polymers.

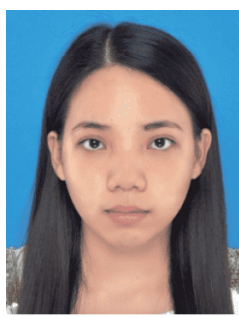

Ziting Tan is currently a PhD student at the School of Nano Technology and Nano Bionics, University of Science and Technology of China, under the supervision of Prof. Liqiang Li. She is currently focusing on the flexible devices, growth of $2 \mathrm{D}$ materials, and interface physical effects of the organic field-effect transistor. 


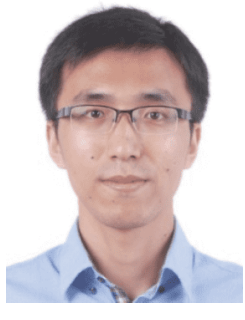

Liqiang Li is currently a full professor at the Institute of Molecular Aggregation Science, Tianjin University. He received his Bachelor's degree (2002) and Master's degree (2005) from Nankai University. He obtained his doctoral degree in physical chemistry (2008) from the Institute of Chemistry, CAS (ICCAS). Then, he worked as a postdoctoral scholar at the Institute of Physics, Muenster University, Germany. In 2014, he joined SINANO, CAS. He moved to Tianjin University in 2019. His research activities concentrate on the assembly, property, and electronic applications of molecular materials (organic semiconductor, conducting polymer, and nano-carbon).

\section{蛋类膜-壳结构启发制备兼具高灵敏度和宽检测 范围的应变传感}

李红卫 ${ }^{1,4 \dagger}$, 谭子婷 ${ }^{1 \dagger}$, 苑力倩 ${ }^{1}$, 李洁 ${ }^{2}$, 陈小松 ${ }^{2}$, 纪德洋 ${ }^{2}$, 张凯 ${ }^{3}$, 胡文平 ${ }^{2}$, 李立强,

摘要 为了解决可拉伸应变传感器的灵敏度与检测范围(最大和最 小可拉伸性)之间相互制约的问题. 受蛋类膜-壳结构的启发, 本文 提出了一种构筑具有高灵敏度、宽可拉伸应变传感器的新策略, 即基于柔性可拉伸的表面接枝聚吡咯膜(s-PPy)(类似于蛋膜)和脆 性金膜(类似于蛋壳)制备器件. Au和s-PPy膜在电学和机械性能上 产生相互协同作用. Au膜在拉伸应变下形成裂纹, 有助于提高应变 传感器的灵敏度并降低其检测极限, 而s-PPy膜桥接Au膜裂纹以增 加器件的可拉伸性, 这一策略在应变传感器中从未使用过. 表面接 枝策略不仅增强界面附着力, 而且使天然脆性的聚吡咯膜表现出 优异的可拉伸性. 在本论文中, 依靠膜-壳互补结构的协同作用, 应 变传感器实现了超高灵敏度(超过 $10^{7}$ ), 宽拉伸范围( $100 \%$ )以及超 低检测极限 $(0.1 \%)$, 这些性能指标将推动应变传感器领域的重大进 步. 而且, 这种基于 $\mathrm{Au} / \mathrm{s}-\mathrm{PPy}$ 膜-壳结构的可拉伸应变传感器可应用 于手指弯曲、手腕旋转、气流波动和声音振动等从微小变形到大 范围变形的应变检测. 以上结果表明基于 Au/s-PPy膜-壳结构的可 拉伸应变传感器具有超高性能和广阔的应用前景. 